\title{
The subject of education in the information society: cognitive identity of a media culture carrier
}

\author{
Iryna Pokulyta, and Mariana Kolotylo* \\ ${ }^{1}$ National Technical University of Ukraine “Igor Sikorsky Kyiv Politechnic Institute”, 37 Peremohy Ave., Kyiv, 03056, Ukraine
}

\begin{abstract}
The article substantiates that the temporality of being of the subject as synchronization of communicative, cognitive demands on a person with dynamic changes of information reality, is the basis for the development of media education. It is justified that the formation of competencies of the carrier of modern culture is a difficult task, since such traits as critical thinking, free possession of judgments about content quality, preventive measures in guarantees of dangers of communicative activity and others, fall into the scope of the main threats - leveling of important conditions of cognitive activity, multiplicity of models of communicative interaction, complex of semiotic systems of media culture, etc. Identified symptomatic cognitive features of the modern subject of information activity indicate the need for an urgent educational response, which means the formation of requirements and criteria for media literacy, a balanced approach to the consideration of temporality, procedural thinking in terms of knowledge, memory, self-identification. It is stated that mastering the semantics, topology, and navigation of the basics of audiovisual communication is an important vector of media education. The article emphasizes the importance of a comprehensive response from the education system to the civilizational challenges of the transition to the information society.
\end{abstract}

\section{Introduction}

Informatization, as enrichment, saturation of the world around us with new and convenient technologies, cannot but please everyone. Informatization of education is no exception. The processes of learning, upbringing are dynamic; there are unprecedented forms, niches and adaptation mechanisms in the organization of the educational segment of culture.

At the same time, the changes of the subject to whom all these efforts are directed are evident. Externally and superficially, the situation looks very positive (it should be noted immediately that we do not include pathological cases, such as Internet addiction, gambling, etc.): modern youth are growing, socialized in the conditions of active use of digital technologies. There can be no other: this is their world, they live in it and carry on the relay of history. Therefore, it is quite logical that young people are adaptable and responsive (unlike the older generation) in obtaining information: applying a minimum of effort, gaining maximum variability, and so on. In addition, generational dissimilarity is a prerequisite for social development, since posterity is the next step in the civilization movement. We also need to capture the fact that the advanced capabilities of the digital technology age are not conferred by institutionalized education. Youth, young people, children underwent their primary socialization as early as the transition of society to the information society. That is, they are shaped by this culture, so further growth of a person through education is primarily characterized by media literacy, a set of competencies, including professional ones. But, thinking about the "further growth of man", it is necessary to understand the present state of things: how fundamental is the dissimilarity of different generations in the aspect of the culture of cognitive activity.

It is important to note that the amount of knowledge in terms of equivalence of the content of modern science is not commensurate with the range of what the educational institutions could have given to their graduates in the recent past. Even these characteristics today are enough to formulate the purpose of the study to elucidate the historical uniqueness of the individuals, who receive educational services. Or else: who is the modern Homo cogitas? What are our perspectives on the relay of history, cultural heritage?

Education as a breeding platform of culture has the same nature as humanity. The very possibility of anthropo-sociogenesis involves, along with other factors, two components of human and social development. It is the education in its heuristic-consolidating project and the image of a person in the demand of the future generation. In other words, evolution in the social context envisaged (today is no exception) the selfreproduction of humanity through the tools of education. The continuity of being is based on the fact that community, and in the case of man, the social community, can continue in the future, embedding the

\footnotetext{
* Corresponding author: kolotylo.mariana@gmail.com
} 
information about the world of culture into the knowledge of the next generation.

The peculiarity of this process is that all factors, including the semantic orientations of the above, are variable and extremely poorly predicted. These are circumstances such as natural conditions (e.g. climatic or other aspects of biogenesis), historical (e.g. external conquests and, as a consequence, assimilation, acculturation, etc.) and many other contexts. Of course, the very content of knowledge is constantly updated. Due to such a fragile, unstable resource for the selfpreservation of mankind, the possibility of evolution (progress) of culture, the appearance of civilizations was and still remains today highly dependent on the whole spectrum of circumstances.

The modern world community has acquired in the course of history a number of significant components of difficult-to-predict development. It is a technodependency, and now a digital extrapolation of culture (both as a convenient prospect and as a new platform for information accumulation). The only one that confronts these challenges of social being is the human cognitive resource. It feeds on the knowledge acquired in the process of education; it is able to convert, modify information into another educational package for future generations.

\section{Literature review, methodology}

The current stage of history - entering the information age, has several features. Outline their basic parameters, stating that information becomes the dominant of different social processes, structures, systems, etc., is insufficient. Researchers such as E. Toffler [1], D. Bell [2], M. Castells [3], etc. addressed the question of characterizing the principles of "postindustrial", "informational" society. Their views had a warning context about the transition to a new type of organization of society, some of which (e.g. the Internet galaxy (M. Castells), the development of virtual communication, the formation of virtual reality) became our reality. Among the various aspects of this without exaggerating global theme, we consider it necessary to emphasize the factor of a new type of information dependency in this kind of public relations. Independence, the insecurity of the subject from the opportunities that reveal information technologies in the external immersion in the personal, even confidential space of the person, have a direct bearing on the lack of media literacy, hence the requirements that are faced with the organization of the media education system.

Increasing the capacity of media literacy is directly related to understanding the specifics of the media communication process. It is, first of all, a study of a new type of communication, drawing on the powerful intelligence of M. McLuhan [4] and L. Manovich [5]. Particular attention needs to be given to the subject's cognitive activity, which includes the need to understand thinking in terms of the laws of logic, starting with Aristotle, the contexts of memory, intuition, subconscious - H. Bergson [6], C. Jung [7], and thinking as creativity $-\mathrm{W}$. Benjamin [8]. It is also necessary to highlight the features of semiotic systems of information interaction. In this article, we focus on the semiotic concept of U. Eco [10].

\section{Problem statement and the aim of the study}

Education in the given civilizational past and present is an institutionalized form for most cultures of the world. Therefore, we can state the social strategies of different societies in their forecasting of the future. In this aspect, it is important to note that, despite the differences in current education models in many countries, the challenges associated with forming a new type of communication, a culture of perception and information processing are common. Exploring new factors in communicative, cognitive, and semiotic directions is the aim of the study. The various challenges need to be addressed separately.

\section{Communicative concepts of media education}

Firstly, it is advisable to consider the creation of a new type of communication. Replaced by the "Gutenberg Galaxy" (M. McLuhan) came electronic media, today "new media" (L. Manovich). Again, at first glance, this is just a change in the way it is transferred - the dissemination of information that should not affect its content. Moreover, broad parameters of the choice of the medium of information and the method (channel) of communication are formed. And, if the New Age is the era of the printed text, then the present is a variability of both visual semantics and audio semantic codes. Information is actively, often very aggressively, through various sensory systems crashing into our consciousness. In the Gutenberg era, being educated, being able to read books was a blessing, a value, a social privilege. At present, this norm of the path to knowledge is being retained, but it is increasingly reminiscent of the nominal one: what is the difference between reading a text or listening to an audiobook, or perceiving a combined digest of information with entertainment elements? In our opinion, there was a communicative atmosphere not only of variability, but also of unstructured semantic flow.

The information made by the wave of history on top of the axiosphere of a globalized society, under the press of its own power, mass, significance, destroys this pyramid of meanings. Pluralism of thoughts and knowledge turns to the absence of a strategy for obtaining them. Along with the depreciation of information, due to its excess at the level of media everyday life, the way to master information is not appreciated. It is illusory to know, not necessarily to remember, even harmful, because tomorrow there will be new information. And if it doesn't (in the case of historical literary works, fundamental discoveries of the past, etc.), there is always a media device, a gadget that will fill the gaps of ignorance. Thus, the modern multimedia type of communication, providing an 
incredible archive of information, reaching the various possibilities of its implementation, eliminated the procedure of comprehension of information.

The latter, in turn, determines the second factor of challenge - the culture of perception and processing of information. In our opinion, its main parameters are the "mosaic" of the communicative environment itself; clickability (not even clipping) as the temporality of the subject's attitude to content and the effect of implantation, the "germination" of the media information space into consciousness and vice versa.

Concerning the third parameter, it is necessary to explain separately what exactly we mean: the deconstruction of the procedure of comprehending information literally consists in the fact that it is melting, as snow, the time to receive it, but this is compensated by the very space of its existence. Thus, dependence is formed in terms of the cognitive function of consciousness in the continuity of nourishment, information saturation. Gradually, the subject loses identification of what he/she knows or does not know, and most importantly, somebody knows, but not him/her. That is, "where?" ends knowledge, principles, beliefs, etc. and "how?" to distinguish, concentrate their own competence.

The practice of educational processes often reveals the following symptom of such a problem: the student as a person, attuned to intellectual activity, occasionally takes his eyes off the smartphone and talks about something. If he/she is stopped and asked to repeat what has been said without the help of the gadget, at best, the last phrases will be reproduced. To present the content of his/her own message becomes an unfulfilled task. Analyzing this symptom, we can make only two assumptions: the first - problem with memory, the second is that the content of the information is irrelevant to the subject, and the information process creates only acoustic noise.

\section{Cognitive dimensions of media literacy}

The topic of "memory" as a philosophical problem is directly related (in the formulation of the question and in the methodology of research) to the cognitive potential of the individual. It is worth pointing out H. Bergson's views on the understanding of the nature of intuition, and the "subconscious" in the theory of psychoanalysis and analytical psychology of C. Jung. Memory, its irreplaceable role, involvement in various manifestations of the entire front of thinking, today falls into the risk zone.

The dependence of the professional, cognitive activity of the person on the amount of knowledge possessed by the person gradually becomes smaller (in other words, "what?" is remembered by the subject). Possession through accessibility, ease of information retrieval, simulates the learned, meaningful knowledge, the value of which does not allow us to relax our cognitive efforts in revitalizing memory structures and mechanisms. Remembering as an activity is offset by knowledge of the roadmap for finding information. Memory does not become a function of consciousness to hold, accumulate, transform the essential parameters of information, but a reservoir for its navigation. That is, the person remembers not the content, but the skills of its search. And what is left to human intelligence: how to create, dream, on what to fantasize, where are the images that nourish our imagination?

The question is rhetorical - in the media space, as an incubator of meanings. It may not be all that sad, but there is clearly a tendency to do so. In the context of this problem, the course of modern education, namely, to teach to learn, does not seem unambiguously successful. On the one hand, this is a necessity because it is extremely difficult to predict future educational preferences. Therefore, the subject must be flexible and adapted to form his own life project of acquiring more and more competences through education, self-education and so on. But today, at least for the above symptoms, loss along the way is noticeable.

The effectiveness of the technological progress of civilization is determined by how much a person is "liberated" from uncreative, monotonous, physically difficult actions through various devices. But along with ease of movement, everyday comfort, etc., modern technology "unloads" the thinking of man, freeing from memory, atrophy the imagination. As for the latter, almost a century ago, W. Benjamin ("A work of art in the age of technical transformability") warned about it. Creativity is not perceived in close connection with creative activity, but only as the ability to achieve what is desired in an unexpected way. Due to the volume, the diverse specification of knowledge, one does not even need to speak about the universal type of personality. It can be a question of integrating knowledge into the modern world, which seems to correlate with the scientific picture, significantly diluted by probabilities, scenarios of reality with virtual character. However, the generation formed one step before the advent of information technology, is forced to combine in their consciousness different civilization platforms. They need to keep in touch basic knowledge with modern requirements of narrow specialization of activity. This means that the mechanisms of critical perception of information still remain (although this does not apply to all older generations and we believe to be directly dependent on education).

For young people, the decision to use this or that information is more often driven by the factors of effort minimization, convenience, the accessibility of the channel to receive it, and the accompanying novelty and entertainment. It is important for students who have a clear purpose in the future profession to apply the knowledge in practice. Although thinking about what theoretical intelligence is behind these empirical techniques is also unnecessary. For the most part, even the motivation to become a unique specialist and to choose the practical options in their professional activity, understanding the causal bases, basic knowledge, does not work. The main features are those that fit into the "Procrustean bed" of competencies. 
Critical thinking is nowadays at the forefront of requirements for future professionals. Based on the properties of critical thinking, it is possible to apply them to the critical understanding of the concept of "critical thinking". Reflecting on the anthropologically determining property of consciousness - the ability to think in the integrity of the active (including practical) attitude of mankind, it is important to note that thinking is the cause and effect; the purpose and means of human existence. The cognitive activity of consciousness in the bowels of the process of anthropogenesis is formed as a holistic mechanism of development. More than a twothousand-year history of studying thinking (beginning with the laws, principles of Aristotle's formal logic), from the point of view of organizing this process for the comprehension of truth, is a difficult way of organizing the most incomprehensible horizons of human desire for ontological meanings.

Scientific discoveries, the creation of essential social development platforms - all this is directly related to the self-reflection of the subject of such activity. It is quite obvious that the order of judgments, the level of understanding - the scale of understanding reality as a sign of this creativity does not imply mass embodiment. Therefore, there are different qualitative levels of thinking, among which one of the highest stages of its development is critical thinking. However, it should be remembered that at its core it is about the integrity of the cognitive system, to climb to the top of which, it is possible only from the plain plateau. Various predicates, such as projective, pre-emptive, transgressive, creative, critical, etc., can only describe an element of a single system. However perfect it may be, this element does not function in isolation from the holistic structure. Therefore, it is necessary to put firstly the task of formation, development of thinking, which in itself cannot be reduced to the invention of the "simulator" of the mind. Or, by choosing a more delicate, imaginative comparison: we are not faced with the choice of Mahayana or Hinayana, the short or long path to truth, and we cannot replace the path by a convenient short way of "transferring" the mind to the "destination".

We make mistakes in the above formal and logical considerations, because we identify the thinking subject with the carrier of consciousness - man. However, what is the independence of judgment, controllability, when critical thinking is considered today in relation to the need to analyze the masses of information materials, a kind of filtering device for various messages, judgments, and ideas. So it is another cognitive process that has the ability to transform reality. Accordingly, its structure is also implanted in the information flows of such a society - the collective consciousness of a globalized network of knowledge, meanings, emotions, values, etc. In this formulation, it is necessary to consider a person as a subject, who produces critical judgments, as they become a means of preventing obstacles to the formation of favorable conditions for the development of a real subject of thought: a global subject of the information society. However, it is also worth noting that this is only a hypothesis that we have a basis for in the scientific research.
Critical thinking, as a cornerstone of contemporary educational requirements, is complemented by media literacy. In parallel with the formation of a new type of communication, there are changes in the way of being in this audiovisual environment. In addition to the advanced communication capabilities, the subject also receives new requirements for his own competencies. Particular attention is paid to the technical side of the issue: the main task is to master modern convenient devices that ensure the status of active users of information networks. Today, the subject has the opportunity, and representatives of the younger generation are realizing it in every possible way, to stay almost without restrictions (time, space) in the system of information contacts through a different kind of media communication platforms. For the older generation, the technical component of the issue sometimes becomes a barrier to this kind of user status in the network communication. However, because of its cognitive nature, this problem is not limited to technical measurement. As users of media communication, we are simultaneously different in terms of tempo-rhythm and saturation of semantic dimensions, which are connected solely by the cognitive structures of our consciousness. In such circumstances, the following question arises: how does it affect the thinking process in the mental, psychological, and identification aspects.

Consider a specific example. When we read a fascinating book, and in the process, someone interferes with the outside, asks about something, then we need some time to "return" to the surrounding reality. We do not even immediately understand what is being said, we do not respond immediately, and so on, because all our attention, cognitive effort, "ontic" (M. Heidegger) immersion was in another semantic reality [9]. Nowadays, the condition of multimedia culture is decentralization and deconstruction of attention. Modern living space is a woven mosaic panel of blocks of different nature, so the very germination of this culture into a personal sense space is at the cost of the loss of a mental, cognitive order. However, it is a current requirement, even a challenge. The educational task in forming media literacy is the answer.

The main trajectory of acquiring media literacy is to train the subject to recognize, classify by quality, and independently create potentially competitive content. Therefore, it is necessary to master the ability to "read" and "write" the whole semantic series contained in the media message, located on the information site, etc. Literacy, as a mastery of the audiovisual semantics of the information space, is one component of success in this activity.

\section{Semiotic context of communication}

Semiosis of media culture is a process that has been going on for more than a century and is conditioned by the emergence of a new type of audiovisual communication, and in recent decades, due to the emergence of "new media" (L. Manovich), its specificity has been modified. In terms of grammar, the semiotic system can be regarded as a sphere of signs, symbols, 
each of which captures a certain value. For example, consider the linguistic system as one of the varieties of the semiotic. Culture is a mechanism of social interaction, having as its component a dialogical basis embodied through a communicative function. Meaningful orientations arise in sociality when they are made out through signal -message -information.

The dialectic of "meaning - thinking" is awakened in the beginnings of human history by the need to stand in solidarity. The information contained in the message, which minimally distorts meaning as an intention, and is accessible to the recipient's perception, already initiates the foundations of the signaling semiotic system. For example, an audio message, such as a signal of danger, because of its naturalness, intonation, acoustic immersion in imitation of sounds of the surrounding world, gradually becomes convenient and accessible by virtue of the anatomical features of the person through communication.

The question of the appearance of language or what type of communication was the first: acoustic, tactile, "audio-tactile" (M. McLuhan) etc., for academic science for obvious reasons (lack of evidence base, impossibility of its reconstruction) has no solution. But the very fact of language creation, relatively speaking the emergence of a "social contract" on the legitimacy of its existence, lays the foundation for development and enables the mode of growth, permanent "replenishment" of the semiotic sphere of culture. Subsequently, there is a need to duplicate a particular message, or rather to capture its meaning in another long-term design: rock drawing pictogram - visual symbol. In the absence of the subject, the message will still reach the addressee.

It is clear that the information potential of these signs was minimal, since knowledge of the decryption code was limited by the number of carriers of this information. The so-called "mimetic semiotic code" (U. Eco) appears. Tens of thousands of years will remain until the "iconic" (U. Eco) code (e.g. writing) appears, but the pictogram is already a second-order sign system. Thus, language and writing, as semiotic varieties of the linguistic communication system, provide an example that communication and information exchange can be carried out in different ways, using separate semiotic systems. In this case, they have different sensory nature.

In parallel with the improvement of communicative "expansion" - the media (M. McLuhan), the semiosphere of culture diversifies and new semiotic systems, such as phonetic writing, emerge. The diversity of cultures and the multidimensionality within one culture affect the process of semiosis in such a way that even a detailed study of them will not cover the entire spectrum of the semiosphere. It can only be stated that the emergence of new social needs, forms of activity, communication methods are dialectically related to the development, functioning of certain semiotic systems. It is especially important to dwell on the difference between mimetic and iconic codes. It should be noted that the advantages of the first is the existing visual path - the link of visual imitation. It allows you to map the subject, thing, action, etc. with their image. Even the imperfect, distant drawing allows us to use this sign (system of signs) without additional grammatical structure - a superstructure of rules. Therefore, literacy as a requirement to use mimetic code is absent (or almost non-existent). In other words, in terms of user requirements, this is the easiest way to convey information.

But there is a downside to the problem: the number of characters. It should be unlimited. Any limitation will mean either at least minimal rules or restrictions on the subject of the image. Regarding the rule-setting option, it is a way of developing a written culture with multiple modifications. In our view, the variant of limitation of the subject of the image is a component of the mimetic code of modern media culture. For example, we all know "emoticons", without which we can no longer imagine the daily conversations with people. Each emoticon represents an emotion, if our feelings are not reduced to one image, we can use several. But, if the situation is not typical, we cannot do without words.

Also in the context of examples of mimetic code, it is important to mention another example - frames (E. Goffman) [11]. The reality itself is framed as a kind of storyboard. On the one hand, it narrows the reality, but it gives the opportunity to cross, balance different communication planes not digital and virtual reality. Thus, mimetic code gains new horizons in contemporary culture for use in media communication. As for the iconic code - the signs of the second order, which have no analogy, resemblance to things, actions, sound, meaning, which encode, then their semiotic capabilities are several orders of magnitude greater. The minimal number of characters combined with the grammar system opens up a huge range of usage. But the condition of their functioning is knowledge of the rules of this grammar. This is also a limitation that can only be overcome by learning the grammatical basis of such a system of signs. An example of a modern icon code is a binary system in programming: zero and one. It is versatile, but proficiency in programming is tied to a deep professional knowledge of applied mathematics. Therefore, we cannot claim that the binary system is the basis of social communication and, accordingly, an element of media literacy.

In the case of semiotic media coverage requirements, it is a matter of knowing, first and foremost, the visual icon code, which is linked to the specifics of various programs, information platforms, which we can use quickly and optimally. This is a visual learning of combined perception, "reading" of the information provided by the frame or interface in combination with the target visual acuity: to see the necessary, decipher the sign and get information among different things.

Another feature of media literacy in the context of mosaic audiovisual culture is the maximum combination of different semiotic codes. Deep, perfect knowledge of only one nowadays limits the subject of activity, both in the communicative everyday life and in the professional spheres of being. For example, at first glance, it is as far from the media profession as a composer - the author of academic music. Classical instruments, live performance, classical acoustic nature of sounds, but now more and more composers (and composers of the 
young generation without exception) create music, using not the usual, many centuries-proven musical notation, but modern computer programs where sound is born in digital system of timbres, bands, musical instruments, etc. This ability requires a special skill: the music that the author's inner hearing hears and touches the piano keys is now being transcribed into the digital version of the composer. With all respect to amateur musicians and amateur versions of this art, however, when it comes to professional authors, performers, it is necessary to point out their training and the requirements of proper possession of this relatively new semiotic system of music.

This version of transcoding the classic profession of artist, architect, is increasingly in need of skills in computer graphics, design, etc. It is not just a tool replacement: it is a computer keyboard and a mouse instead of a paintbrush and paint. It is a change in the semiotic system that is complicated and simplified at the same time. New grammar is emerging - a second-order sign, so the movement goes from mimetic to iconic code. And as discussed above, it broadens the range of possibilities and at the same time narrows the range of people who possess this knowledge. In architecture, it is an opportunity to travel the space that previously existed only in the imagination of the author. People had to wait for the building to be created and only then could the architect's design be imagined. Current technologies, virtual projects make it possible to carry out a kind of testing of the building before this building will either be a city decoration or change its appearance for many years.

We have given examples of the arts professions, in which the special requirement of media literacy is mastering a new communication system. However, there are examples of industries in which the profession is receiving a different semiotic, communication basis, and their number is increasing in different areas of activity. Such sciences as theoretical physics, microbiology, etc. do not need optical devices of their own development and discoveries. With the help of media - computer programs, virtual reality is simulated a world that anyone can look into. It enormously enriches our ideas about the universe, creates a media picture of the world, which acts as a kind of compensation for those knowledge and ideas that each of us cannot get in full because of their incredible scale and industry specificity.

The organizing specificity of mimetic and iconic codes in the formation of modern media communication is also considered by another author - Ch. Gere [12], but in the aspect of screen culture. The screen itself today acts as a certain topos - the place of our worldview, cognitive, leisure existence. And despite the physical size of a particular screen - it actually unfolds the panorama of the media world, which prevails informally in relation to the surrounding non-screen reality. This in turn means that the frame, the boundary of this reality, we are gradually learning not to notice. The behind-thescenes communicative space embraces us wholeheartedly, and we are sensory focused on the media image.
The world around is dimming not only in spectacular but also in temporal and spatial features. But screen magic has, according to the author, its nature - the genealogy of visual communication. Rectangular, square, oval - various forms of the image, which are locked inside a geometric figure, have passed their approbation in the history of fine arts. Thus, the visual culture of modern civilization incorporates historically formed "screen" models, focusing on two main types: "portrait" and "landscape", each of which corresponds to a corresponding semiotic code, semantic load and communicative environment.

The semiosis of the contemporary audiovisual media culture is based on the factors of information society development discussed above. First of all, it is an easyto-identify and interactive sign system. There is also a semiotic series of characters that carry an emotional and psychological color and have an anthropomorphic imprint. The verbal segment of information remains important, but for the most part it does not play a central role. A common feature of the semiotic ordering of the message is the compositional scheme of information blocks scattered in the screen plane. The visual topology corresponds to a meaningful navigator of the information space. Today, a large number of specific audiovisual codes have been developed. Awareness of this issue, in our opinion, is a significant part of formation of media literacy.

\section{Conclusions}

All the issues considered point to the tendencies of cultural transformations in connection with the new communication strategies as the civilizational value of the formation of the information society. This transition makes considerable demands on the subject in terms of mastering the competencies of the carrier of the modern way of human existence. The main attention was paid to the dangers that accompany changes in the ontic characteristics of media culture: cognitive, mental etc. The process of thinking: new tempo-rhythmics, speed and quality of processing, evaluation of information, creation of competitive content; sensory experience: audiovisual defocus, at the same time targeting perception; semiotic polyphony: depth of ownership and, at the same time, variability in the use of different in nature and functions of the screen codes of media communication - all of them are the main guidelines of the named cognitive, mental and identification factors of formation of the subject of the modern educational process.

However, the material discussed above concludes that the learning process today cannot be guided by these characteristics, such as skills, knowledge, competencies, that are amenable to training or isolated development in the context of different educational tasks. If these media literacy requirements are the subject of training, then we are encroaching, even destroying the subjectivity itself. Therefore, it is necessary to create a semantic vertical - a symphonic score of fostering a mentally whole selfsufficient personality. Therefore, in our opinion, the content of modern education, in particular media education, should be unprecedented approaches in which 
information resources, social goals and means of their implementation are based on preserving the identity of the subject. The person is tasked not only to fit into the information reality of the present, but also to acquire knowledge, the ability to actively build their own semantic space.

Therefore, the development of media education, the depth of awareness of the problems and the development of methods, approaches to ensure the media literacy of the subject - the carrier of the culture of information society, is the basis of self-preservation of human civilization.

\section{References}

1. A. Toffler, The Third Wave (Bantam Books, New York, 1980)

2. D. Bell, The coming of the post-industrial society: a venture in a social forecasting (2011), https://www.os3.nl/_media/2011-2012/daniel_bell_the _coming_of_post-industrial_society.pdf. Accessed 15 November 2019

3. M. Castells, The Internet galaxy: reflections on the Internet, business and society (Oxford University Press, Oxford, 2003)

4. M. McLuhan, The Gutenberg Galaxy: The Making of Typographic Man (University of Toronto Press, Toronto, Buffalo, London, 2011)

5. L. Manovich, The language of new media (2001), https://dss-edit.com/plu/ManovichLev_The_Language_of_the_New_Media.pdf. Accessed 28 December $201 \overline{9}$

6. A. Bergson, Tvorcheskaya evolyutsiya (Creative Evolution). (Harvest, Minsk, 1996)

7. K. Yung, Arkhetip i simvol (The archetypes and the collective unconscious). (Renessans, Moscow, 1991)

8. W. Benjamin, The Work of Art in the Age of Mechanical Reproduction, in Illuminations, ed. by H. Arendt (Schocken New York Books, 1969)

9. M. Khaydegger, Bytiye i vremya (Being and Time). (Folio, Kharkov, 2003)

10. U. Eko, Otsutstvuyushchaya struktura. Vvedeniye v semiologiyu (The absent structure: Introduction to Semiotics). (Petropolis, Saint Petersburg, 1998)

11. E. Goffman, Frame Analysis: An Essay on the Organization of Experience (Northeastern University Press, Boston, 1986)

12. Ch. Gere, Visual Communications 5(2), 141-152 (2006) 